\title{
Fully tunable active polarization imager for contrast enhancement and partial polarimetry
}

\author{
Guillaume Anna, ${ }^{1}$ Hervé Sauer, ${ }^{1}$ François Goudail, ${ }^{1, *}$ and Daniel Dolfi' \\ 'Laboratoire Charles Fabry, Institut d'Optique, CNRS, University Paris Sud 11, \\ 2, Avenue Augustin Fresnel, 91127 Palaiseau, France \\ ${ }^{2}$ Thales Research and Technology_France, RD128, 91767 Palaiseau Cedex, France \\ ${ }^{*}$ Corresponding author: francois.goudail @ institutoptique.fr
}

Received 28 March 2012; revised 29 May 2012; accepted 3 June 2012; posted 6 June 2012 (Doc. ID 165677); published 20 July 2012

\begin{abstract}
We present the design and the practical implementation of a polarimetric imaging system based on liquid-crystal modulators that allows generation and analysis of any polarization state on the Poincaré sphere. This system is more versatile than standard Mueller imagers that are based on optimized, but limited, sets of illumination and analysis states. Examples of benefits brought by these extra degrees of freedom are illustrated on two different applications: contrast enhancement and extraction of partial polarimetric properties of a scene. (C) 2012 Optical Society of America

OCIS codes: $110.5405,110.4280$.
\end{abstract}

\section{Introduction}

Polarimetric images are useful for gathering information that is not visible on intensity images. They have many applications in machine vision, remote sensing, biomedical imaging, and industrial control [1-3]. In particular, several types of Mueller imaging systems have been designed and demonstrated [4-6]. Such imagers illuminate the scene with four different polarization states and analyze the light diffused by the observed scene along four different states. By acquiring 16 images with 16 combinations of illumination/analysis states, they can measure the whole Mueller matrix of each pixel of the scene. Their illumination and analysis states are in general optimized to reduce the propagation of errors during the inversion process that leads from the measurements to the estimated Mueller matrix [7-9], and the systems are calibrated to compensate for the unavoidable discrepancies between the theoretical and real characteristics of the components [10].

$1559-128 \mathrm{X} / 12 / 215302-08 \$ 15.00 / 0$

(C) 2012 Optical Society of America
Although Mueller imaging has demonstrated its efficiency in many applications [11-13], there are cases in which measuring the whole Mueller matrix is not necessary. For example, if the purpose is to optimize discrimination between a target and an object of interest, it has been demonstrated that a limited number of images is sufficient [14-17]. However, these images have to be acquired with illumination and analysis states that depend on the scene and may lie anywhere on the Poincaré sphere. In other cases, it may be sufficient to extract only partial information, such as particular coefficients or set of coefficients of the Mueller matrix $[18,19]$. In such applications, the fixed states adapted to acquisition of the whole Mueller matrix may not be optimal for acquiring the required information with a minimum number of measurements.

To address this issue, we have designed and implemented an experimental setup, based on liquidcrystal variable retarders, that makes it possible to illuminate the scene and analyze the light scattered by the scene in any polarization state. In Section $\underline{2}$ we will first describe this setup, its performance, and its limits. Then in Section 3 we will show its benefits in two different domains of application: contrast 
optimization for target detection, and extraction of polarimetric properties with a minimal number of acquisitions and optimal precision.

\section{Design and Implementation of the Polarimetric Imager}

The active polarimetric imaging system that we wish to realize is based on the following principle. The scene is illuminated with light whose polarization state is defined by a Stokes vector $\mathbf{S}$ and is produced by a polarization state generator (PSG). The polarimetric properties of a region of the scene corresponding to a pixel in the image are characterized by its Mueller matrix $M$. The Stokes vector of the light scattered by this region is $\mathbf{S}^{\prime}=M \mathbf{S}$. It is analyzed by a polarization state analyser (PSA), which is a generalized polarizer allowing selection of photons characterized by the Stokes vector T. The number of photoelectrons measured at a pixel of the sensor is

$$
i=\frac{\eta \tau I_{0}}{2} \mathbf{T}^{T} M \mathbf{S}
$$

where the superscript $T$ denotes matrix transposition. In this equation, $\mathbf{S}$ and $\mathbf{T}$ are Stokes vectors representing unit intensity, purely polarized light $\left(\mathbf{S}=\left[s_{0}, s_{1}, s_{2}, s_{3}\right]^{T}, s_{0}^{2}=s_{1}^{2}+s_{2}^{2}+s_{3}^{2}=1\right.$, and similarly for T), $I_{0}$ (in number of photons) is the photons flux illuminating the area of the scene associated with a pixel of the camera, $\tau$ is the transmission coefficient associated with all the optical elements of the system, and $\eta$ is the conversion efficiency between photons and electrons.

This setup constitutes the building block of a large number of polarimetric imaging systems [20-22]. Indeed, by using a fixed illumination state and four different analysis states, one obtains a Stokes image, and by using 16 different combinations of illumination and analysis states, one can measure the whole Mueller image. However, these imaging setups are optimized to measure Stokes and/or Mueller data, and they can generate or analyze only four fixed polarization states. They are, in general, not able to generate and analyze arbitrary polarization states on the Poincaré sphere. In this section, we describe an imaging system having this capability.

\section{A. Description of the Imaging Setup}

The active polarimetric imager that we have built is represented in Fig. 1. The illumination part is composed of a white-light fiber source $(\mathrm{Fs})$ followed by a spotlight (Sl) (from LINOS), allowing us to reduce the numerical aperture at the output of the fiber (from $70^{\circ}$ to $15^{\circ}$ in our case). A diffuser is used to completely homogenize the beam in the plane of the lens $\mathbf{L}_{\mathbf{1}}$. Using two lenses $\mathbf{L}_{1}$ and $\mathbf{L}_{2}$, we design a Khöler illumination that allows us to illuminate uniformly and with a small numerical aperture the polarization state generator composed of one polarizer $\left(\mathbf{P}_{1}\right)$ and two liquid-crystal variable retarders $\left(\mathbf{L C}_{\mathbf{1 , 2}}\right.$, from Meadowlark Optics). This design is necessary since the liquid-crystal cells have a critical acceptance angle. If the incidence angle was too large, the polarization in the field could be different from that on the axis, which would be very damaging for our application. Thanks to this setup, the beam modulated by the PSG is uniform in both intensity and polarization. It is then sent to infinity by a third lens $\left(\mathbf{L}_{\mathbf{3}}\right)$ to illuminate the scene. The field diaphragm (FD) allows control of the size of the beam on the object, and the aperture diaphragm (AD), control of the intensity of the illumination beam.

The light backscattered by the scene is then analyzed with a PSA composed, as the PSG, of two liquid-crystal variable retarders $\left(\mathbf{L C}_{\mathbf{3 . 4}}\right)$ and one polarizer $\left(\mathbf{P}_{2}\right)$. The PSA is followed by a spectral filter with a center wavelength of $640 \mathrm{~nm}$ and $10 \mathrm{~nm}$ bandwith. The bandwidth has to be small due to the dependence of the retardance on the wavelength. Finally, the photons passing through the filter are collected by a 14 bit CCD camera (C) that produces an intensity image.

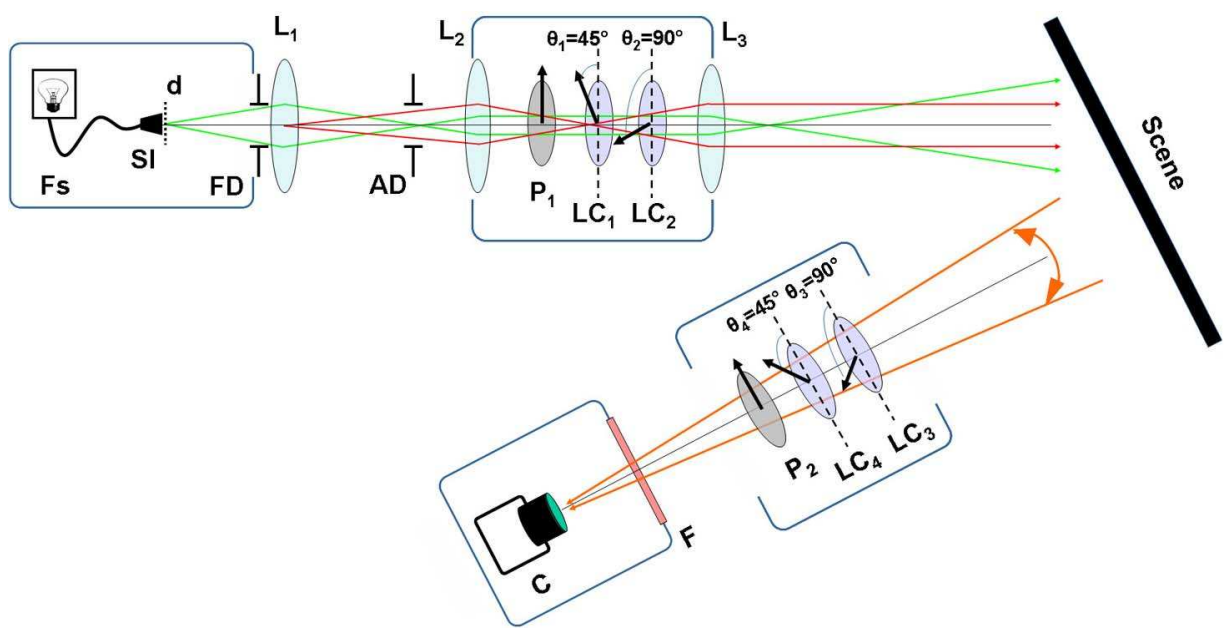

Fig. 1. (Color online) Scheme of the imaging system: white-light fiber source; Sl, spotlight reducing the aperture angle at the output of the fiber; d, diffuser; FD, field diaphragm; $\mathrm{AD}$, aperture diaphragm; $\mathbf{P}_{\mathbf{1}}, \mathbf{P}_{\mathbf{2}}$, polarizers; $\mathbf{L} \mathbf{C}_{\mathbf{1}, \ldots, \mathbf{4}}$, liquid crystals; F, spectral filter; C, camera. 
Considering the choice of the PSG and the PSA, it has to be noticed that different ways exist to generate all the polarization states on the Poincaré sphere. They depend on the components used to control the polarization. In our case, we have chosen liquidcrystal variable retarders (LCVR) that are particularly well suited for polarimetric imaging. Indeed, they have a good acceptance angle and offer wide, clear aperture, nearly no aberrations, and no image wander. When using such components, different configurations can be considered to solve our problem [23-25]. We have chosen the configuration represented in Fig. 2. It consists of one polarizer followed by two LCVR that are oriented respectively with angles $\gamma$ and $\theta$ with respect to the axis $x$ defined by the direction of the linear polarizer.

It has been shown that only the configurations associated with angles $\gamma= \pm 45^{\circ}$ and $\theta=\gamma \pm 45^{\circ}$ allow generation of any polarization state on the Poincaré sphere [24], and this is why, in our experiment, the angles $\gamma$ and $\theta$ are equal, respectively, to $45^{\circ}$ and $90^{\circ}$.

\section{B. Experimental Validation}

We controlled the performance of the PSG and the PSA with a commercial polarimeter and verified the agreement between the nominal polarization states and those actually generated by the system. To quantify the difference between the actual polarization states and those theoretically expected, we use the angle separating these two states on the Poincaré sphere. The angle $D$ between two polarization states with respective azimuth $\left(\alpha_{1}, \alpha_{2}\right)$ and ellipticities $\left(\varepsilon_{1}, \varepsilon_{2}\right)$ is defined by

$$
\begin{aligned}
D= & \cos ^{-1}\left[\sin \left(2 \varepsilon_{1}\right) \cdot \sin \left(2 \varepsilon_{2}\right)\right. \\
& \left.+\cos \left(2 \varepsilon_{1}\right) \cdot \cos \left(2 \varepsilon_{2}\right) \cdot \cos \left(2\left(\alpha_{1}-\alpha_{2}\right)\right)\right] .
\end{aligned}
$$

We generated 16 polarization states distributed over the whole Poincaré sphere, and we obtained the results presented in Fig. $\underline{3}$. The maximal angle between the experimental and theoretical polarization states, on the Poincaré sphere, is about $4.5^{\circ}$, which represents a maximal error of $2.25^{\circ}$ in azimuth or ellipticity. This precision is sufficient for the applications that we will address in the following section.

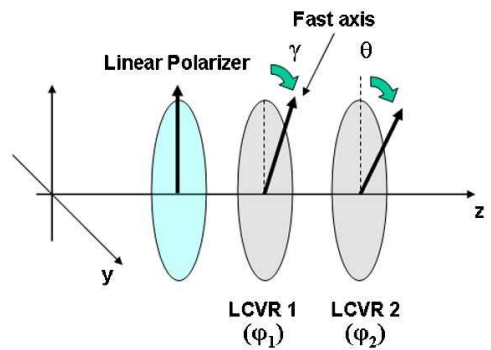

Fig. 2. (Color online) Scheme of a PSG consisting of one linear polarizer followed by two fixed retarders. The orientations of the fast axes of the two variable retarders are given by the angles $\gamma$ and $\theta$ with respect to the direction of the polarizer. $\phi_{1}$ and $\phi_{2}$ are the phase shifts introduced respectively by LCVR 1 and 2 .

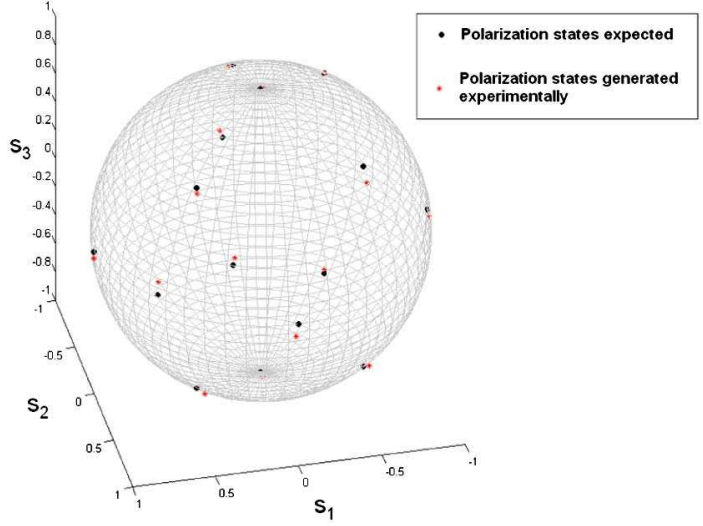

Fig. 3. (Color online) Comparison between the polarization states theoretically expected and effectively generated using the PSG presented Fig. 2 with the angles $\gamma=45^{\circ}$ and $\theta=90^{\circ}$

If better precision was needed, different sources of errors should be compensated for. First is the mechanical positioning of the liquid crystals, because if they are not oriented at their nominal angles, the applied voltage does not generate the expected polarization states. In our case, we did not use very precise mechanisms to orient the crystals, which could be the source of some systematic errors. The second potential source of error is the temperature dependence of the liquid crystal response. Indeed, the response of LCVR strongly depends on the temperature, and even if our cells are controlled in temperature, small fluctuations can appear and lead to random errors.

\section{Examples of Applications}

Polarization contains valuable information that can be helpful for enhancing imaging performance. Many active polarimetric imaging systems already exist, but the set of illumination and analysis states that they can implement is usually limited. Our purpose in the present section is to illustrate the benefit of being able to generate and analyze any polarization state on the Poincaré sphere. We will give two examples: contrast optimization for target detection and extraction of partial polarimetric properties such as subsets of Mueller matrix coefficients.

\section{A. Contrast Enhancement}

Contrast enhancement is an important research field in the domain of imaging. In the radar domain, polarization has been used for a long time to enhance the contrast in synthetic aperture radar (SAR) images [26]. More recently, polarization has also been proven useful for enhancing the contrast in optical imaging $[22,27,28]$. However, standard polarimetric imaging systems may not be able to reach the highest possible value of the contrast for two main reasons. The first one is that these systems, as they can only use a limited number of polarization states, are not able to fully adapt to the scene or to the measurement conditions. The second reason is that they often need to acquire multiple images to 
extract the relevant information (e.g., at least, 4 images in a Stokes imaging system, or 16 images in a Mueller imaging system). Recently, it has been theoretically demonstrated that when considering detection of a single target of interest against a background, the maximal value of the contrast can be reached by a single measurement, but this measurement has to be performed with optimized PSG and PSA states [29]. Of course, these optimal states depend on the scene and may be located anywhere on the Poincaré sphere. Using the system that we have built, we will present in the following experimental results that confirm this theoretical expectation.

The image in Fig. 4(a) is the Mueller image of a scene composed of some polygonal chunks of sandpaper glued on a sandpaper of different roughness, the whole scene being covered with the same paint. By looking at the $M_{00}$ image, which corresponds to an intensity image of the scene, we can see that the objects and the background cannot be discriminated. We consider in this experiment that the total integration time to perform the acquisition is kept constant and equal to $t_{0}$. Since 16 images have to be acquired to compute the entire Mueller matrix, the integration time used for the acquisition of each image was about $t_{0} / 16$. On the other hand, Fig. 4(b) represents the image obtained with PSG and PSA states that maximize the contrast between two types of materials $[16,28]$. This single image has been acquired with an integration time of $t_{0}$, which explains why
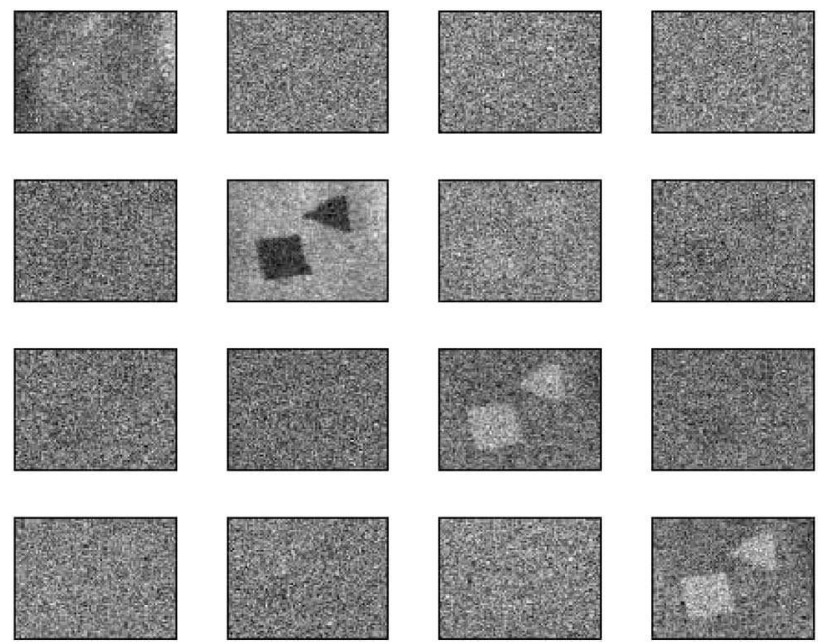

(a) Mueller matrix of the scene

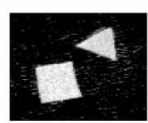

(b) Optimal image $\left(80^{\circ},-10^{\circ}\right)\left(15^{\circ},-10^{\circ}\right)$

Fig. 4. (a) Mueller matrix of a scene composed of some polygonal chunks of sandpaper glued on a sandpaper of different roughness, the whole scene being cover with the same paint. The integration time for the acquisition of each image used to compute the Mueller matrix is $t_{0} / 16$. (b) Optimal image obtained by using the set of polarization states in illumination and analysis maximizing the contrast. The integration time for the acquisition is $t_{0}$. it is much less noisy than the Mueller images. We also observe that the contrast has been significantly improved by using an optimal couple of polarization states in illumination and analysis.

To quantify the contrast improvement, we will use the Bhattacharyya distance [21] associated with $N$ dimensional data:

$$
\begin{aligned}
\mathcal{B}_{t, b}= & \frac{1}{8}\left(\bar{x}_{t}-\bar{x}_{b}\right)^{T}\left[\frac{\Gamma_{t}+\Gamma_{b}}{2}\right]^{-1}\left(\bar{x}_{t}-\bar{x}_{b}\right) \\
& +\frac{1}{2} \log \left[\frac{\operatorname{det}\left(\frac{\Gamma_{t}+\Gamma_{b}}{2}\right)}{\sqrt{\operatorname{det}\left(\Gamma_{t}\right) \operatorname{det}\left(\Gamma_{b}\right)}}\right],
\end{aligned}
$$

where $\bar{x}_{u}$ is a vector of dimension $N$ containing the average values associated with the region $u=\{t, b\}$ (respectively, target and background) in each dimension and $\Gamma_{u}$ is the associated covariance matrix. In the case where we acquire the full Mueller matrix, the data are a set of $N=16$ images associated with each coefficient of the matrix. In the case where we acquire only the optimal image, the data consist of only $N=1$ intensity image.

The Bhattacharyya distances associated with the entire Mueller image [Fig. 4(a)] and the optimal image [Fig. $\underline{4(\mathrm{~b})}$ ] are the following:

$$
\mathcal{B}_{\text {mueller }}=4.6, \quad \mathcal{B}_{\text {opt }}=18.6 .
$$

As expected, the contrast is much larger using only one optimized acquisition than acquiring the entire Mueller matrix if we consider that we have a fixed amount of time to observe the scene.

This first example shows the improvement obtained by using optimized polarizations that capture in a single image all the information useful for discrimination. However, this application requires the knowledge of the polarimetric properties of the observed scene. A further question is thus: Is it possible to use our system to extract quickly the polarimetric properties required to enhance the contrast? We bring a possible solution to this issue in the next section.

\section{B. Extraction of Mueller Matrix Coefficients}

To extract all the polarimetric properties of an object, we need to acquire the full Mueller matrix, which requires the acquisition of 16 images. However, we have to note that in some cases, physical constraints can reduce the number of degrees of freedom of the Mueller matrix. These constraints might be, for example, knowledge that the scene does not possess certain properties (such as birefringence), making some Mueller matrix elements zero. It is thus interesting to develop a protocol to measure only the nonzero coefficients of the matrix, which would require less than 16 measurements and thus decrease the acquisition time.

It is well known that by using four polarization states located at the vertices of a regular tetrahedron 
on the Poincaré sphere for lighting and analyzing, it is possible to minimize the propagation of errors during the estimation of the Mueller matrix from the measured data [9]. However, this method is optimal for extracting the entire Mueller matrix. If less than 16 coefficients have to be estimated, it may possible to further improve estimation performance by using a reduced number of acquisitions with optimized couples of illumination/analysis states. It is thus necessary to determine which couples of polarization states allow extraction of the desired coefficients of the Mueller matrix with optimal precision. This issue has been addressed theoretically in such works as $[19,30]$. We will, in the following, investigate how a polarimetric imager with full degrees of freedom can further improve acquisition of partial polarimetric information.

\section{Model for Multiple Polarimetric Image Acquisition}

To extract polarimetric information from a scene, an active polarimetric imager will perform a number $N$ of intensity measurements in the following way:

$$
\mathbf{T}_{n}^{T} M \mathbf{S}_{n}=I_{n} ; \quad n \in\{1, \ldots, N\},
$$

where $\mathbf{S}_{n}$ denotes the PSG Stokes vector, $\mathbf{T}_{n}$ denotes the PSA Stokes vector, $M$ denotes the Mueller matrix of the observed pixel, and $I_{n}=2 i_{n} /\left(\eta \tau I_{0}\right)$ [see Eq. (1)]. It has to be noted that the value of the intensity $I_{n}$ depends on four parameters $\left(\alpha_{n}^{S}, \varepsilon_{n}^{S}\right)$ and $\left(\alpha_{n}^{T}, \varepsilon_{n}^{T}\right)$ that are respectively the azimuth and the ellipticity of the polarization states used in illumination and analysis:

$$
\begin{aligned}
& \mathbf{S}_{n}\left(\alpha_{n}^{S}, \varepsilon_{n}^{S}\right)= {\left[\begin{array}{c}
1 \\
\cos \left(2 \alpha_{n}^{S}\right) \cos \left(2 \varepsilon_{n}^{S}\right) \\
\sin \left(2 \alpha_{n}^{S}\right) \cos \left(2 \varepsilon_{n}^{S}\right) \\
\sin \left(2 \varepsilon_{n}^{S}\right)
\end{array}\right] } \\
& 1 \\
& \mathbf{T}_{n}\left(\alpha_{n}^{T}, \varepsilon_{n}^{T}\right)=\left[\begin{array}{c}
\cos \left(2 \alpha_{n}^{T}\right) \cos \left(2 \varepsilon_{n}^{T}\right) \\
\sin \left(2 \alpha_{n}^{T}\right) \cos \left(2 \varepsilon_{n}^{T}\right) \\
\sin \left(2 \varepsilon_{n}^{T}\right)
\end{array}\right],
\end{aligned}
$$

with $\alpha_{n} \in[-\pi / 2, \pi / 2]$ and $\varepsilon_{n} \in[-\pi / 4, \pi / 4]$.

Let us first consider that $N=16$. In this case, one can access the entire Mueller matrix, and Eq. (5) is equivalent to the vector-matrix product:

$\forall n \in\{1, \ldots, 16\}, \quad \mathbf{W}_{n}^{T} \mathbf{V}_{M}=I_{n} \Leftrightarrow Q_{T, S} \mathbf{V}_{M}=\mathbf{I}$,

where $\mathbf{V}_{M}$ is the 16-component vector obtained by reading the Mueller matrix $M$ in the lexicographic order, $\mathbf{W}_{n}$ the 16-component vector computed from the Kronecker product $(\otimes)$ between the illumination and analysis states $\left(\mathbf{W}_{n}=\mathbf{T}_{n} \otimes \mathbf{S}_{n}\right.$ with $n \in\{1, \ldots, 16\}), Q_{T, S}$ the $16 \times 16$ square matrix obtained by stacking the vectors $\mathbf{W}_{n}^{T}$ row by row, and
I the vector obtained by stacking the intensity values $I_{n}$.

From Eq. (7), it is possible to extract the elements of the Mueller matrix using the inverse of the $16 \times 16$ square matrix $Q_{T, S}$ :

$$
\mathbf{V}_{M}=Q_{T, S}^{-1} \mathbf{I} .
$$

Several authors have tried to optimize the condition number of the matrix $Q_{T, S}$ to minimize error propagation during this inversion $[19,30]$. Indeed, the condition number of a matrix measures the sensitivity of the solution of a system of linear equations to errors in the data. It gives an indication of the accuracy of the results from matrix inversion. It is given by the ratio of the largest singular value of the matrix to the smallest:

$$
\operatorname{cond}(Q)=\frac{\sigma_{\max }}{\sigma_{\min }},
$$

where $\sigma_{\max }$ and $\sigma_{\min }$ denote, respectively, the largest and smallest singular values of the matrix $Q$. A "wellconditioned" matrix is thus associated with a value near 1 , while a "badly conditioned" matrix is associated with a high value of the condition number. If this latter is equal to infinity, it means that at least one of the singular values of the matrix is equal or almost equal to zero, and thus that the matrix is singular.

\section{Optimal Extraction of Partial Polarimetric} Information

Let us now assume that some coefficients of the Mueller matrix under investigation a equal to zero. The nonzero coefficients of this matrix form a set $\Omega$ of cardinal $N_{\Omega}$. Let us take an example:

$$
M=\left[\begin{array}{cccc}
M_{00} & M_{01} & 0 & 0 \\
0 & M_{11} & M_{12} & 0 \\
0 & M_{21} & M_{22} & 0 \\
M_{30} & 0 & 0 & M_{33}
\end{array}\right]
$$

In this case, the set $\Omega$ containing all the indices of the nonzero Mueller matrix coefficients is $\Omega=$ $\{00,01,11,12,21,22,30,33\}$ and $N_{\Omega}=8$. Our purpose will be to measure these nonzero coefficients with optimal precision by using an optimal association of a number $N_{\Omega}$ of PSG/PSA (S, T) couples. Taking into account the fact that only the coefficients in $\Omega$ are nonzero, Eq. (7) can be written as

$$
Q_{T, S}^{\Omega} \mathbf{V}_{M}^{\Omega}=\mathbf{I}^{\Omega},
$$

where $\mathbf{V}_{M}^{\Omega}$ is a $N_{\Omega}$-dimensional vector containing all the nonzero coefficients of the Mueller matrix, $Q_{T, S}^{\Omega}$ is the $N_{\Omega} \times N_{\Omega}$ matrix whose lines are vectors $\mathbf{W}_{n}$ [see Eq. (7)], from which only the $N_{\Omega}$ coefficients indexed by the set $\Omega$ are kept, and $\mathbf{I}^{\Omega}$ is the vector 
obtained by stacking the $N_{\Omega}$ intensity values obtained.

To minimize the error propagation during inversion, the matrix $Q_{T, S}^{\Omega}$ has to have a condition number as close as possible to unity. Our goal is thus to find the best association of PSG/PSA yielding a matrix $Q_{T, S}^{\Omega}$ with a minimal condition number:

$$
(\mathcal{S}, \mathcal{T})=\arg \min _{\left(\mathbf{S}_{n}, \mathbf{T}_{n}\right), n \in\left\{1, \ldots, N_{\Omega}\right\}}\left\{\operatorname{cond}\left[Q_{T, S}^{\Omega}\right]\right\},
$$

where $\mathcal{S}$ and $\mathcal{T}$ are respectively the sets of PSG $\left(\mathbf{S}_{n}\right)$ and PSA $\left(\mathbf{T}_{n}\right)$ with $n \in\left\{1, \ldots, N_{\Omega}\right\}$ that minimize the condition number of the $Q_{T, S}^{\Omega}$ matrix.

To perform this search, one has to pay attention to the number of parameters that have to be optimized simultaneously. Indeed, each intensity image depends on four parameters, e.g., the azimuth and ellipticity of the PSG and PSA states. Optimizing the $N_{\Omega}$ PSG/PSA states needed for the calculation thus involves $4 \times N_{\Omega}$ parameters, which can be quite large. Moreover, it is likely that the performance criterion will have local minima. It is thus necessary to use an algorithm robust to the presence of local maxima. After comparing different solutions, we have chosen to use the shuffled complex evolution (SCE-UA) method [31]. This algorithm consists in generating different sets of illumination and analysis polarization states and in changing them by using a global evolution framework that finally leads to a parameter set given a well minimized condition number of the $Q_{T S}^{\Omega}$ matrix.

To validate our method, we first look for four polarization states that can be used in both illumination and analysis to extract the entire Mueller matrix (this represents an optimization on eight parameters). In this case, it is already known that the minimal value of the condition number is 3.00 , and that this value is reached when the four polarization states form a regular tetrahedron on the Poincaré sphere [32,33]. Our algorithm actually reaches a solution having these properties. If we now wish to optimize all 16 PSG/PSA couples simultaneously (which represents optimization on 64 parameters), the algorithm converges toward a minimal value of the condition number of 3.8, close to the best one expected, but a little different, certainly due to the fact that the difficulty of reaching a global minimum grows with the number or parameters to optimize.

We will now illustrate the improvement in estimation precision brought by optimizing the PSG and the PSA configuration for $N_{\Omega}$ measurements with $N_{\Omega} \leq 16$. The first example is a comparison of the results of our approach and those presented in a recent paper for the extraction of polarimetric coefficients using a reduced set of optimized images. The second is an experimental validation of the concept in a real-world example.

\section{Optimizing the Acquisition of Specific Mueller Matrices}

In a recent paper, Savenkov and al. [19] presented a way to optimally extract the nonzero components of a block-diagonal Mueller matrix of the form

$$
M=\left[\begin{array}{cccc}
M_{00} & M_{01} & 0 & 0 \\
M_{10} & M_{11} & 0 & 0 \\
0 & 0 & M_{22} & M_{23} \\
0 & 0 & M_{32} & M_{33}
\end{array}\right]
$$

using only eight acquisitions. This interesting result has been obtained by optimizing the PSG configurations and using the Stokes imaging system in analysis. The optimization is thus only done on the polarization states in illumination, and it is likely that the results could be further improved by optimizing the illumination and analysis states simultaneously, which is possible with our imaging system. We have thus applied our optimization method to this problem. Simultaneous optimization of the eight PSG/PSA configurations allowing extraction of the eight nonzero coefficients of the Mueller matrix with the best condition number yields the result presented in Table 1 .

The condition number of the associated $Q_{T, S}^{\Omega}$ matrix is equal to 2.70. Let us now compare these results with those obtained with Stokes imaging. We consider that this Stokes imaging system uses the four optimal states that allow minimization of errors due to propagation of the noise when inverting the measures [9]. According to [19], the two optimal PSG states are $\left(79.8^{\circ}, 14.7^{\circ}\right)$ and $\left(-42.5^{\circ}, 37.6^{\circ}\right)$ (azimuth and ellipticity). The condition number of the matrix $Q_{T, S}^{\Omega}$ associated with these states is 6.9. It is more than twice larger than the result obtained by optimizing simultaneously the illumination and analysis states.

However, it is difficult to precisely relate condition numbers with actual processing performance. To have a more concrete understanding of the performance improvement, we will use the following criterion:

$$
\mathcal{C}=\operatorname{trace}\left[\left(\left[Q_{T, S}^{\Omega}\right]^{T} Q_{T, S}^{\Omega}\right)^{-1}\right]
$$

Table 1. Set of Optimal States in Illumination and Analysis, Respectively, of Azimuth And Ellipticity $\left(\alpha_{s}, \varepsilon_{s}\right),\left(\alpha_{t}, \varepsilon_{t}\right)$, Minimizing the Condition Number of the $Q_{T, S}^{\Omega}$ Matrix in the Case of a Mueller Matrix of the Form in Eq. (13)

\begin{tabular}{lcc}
\hline Image $n^{\circ}$ & $\left(\alpha_{s}, \varepsilon_{s}\right)$ & $\left(\alpha_{t}, \varepsilon_{t}\right)$ \\
\hline 1 & $\left(-56.4^{\circ}, 8.1^{\circ}\right)$ & $\left(60.8^{\circ},-6.3^{\circ}\right)$ \\
2 & $\left(2.35^{\circ}, 43.0^{\circ}\right)$ & $\left(-49.6^{\circ},-4.2^{\circ}\right)$ \\
3 & $\left(49.8^{\circ}, 5.7^{\circ}\right)$ & $\left(53.3^{\circ}, 17.7^{\circ}\right)$ \\
4 & $\left(-72.4^{\circ},-7.1^{\circ}\right)$ & $\left(5.4^{\circ},-5.1^{\circ}\right)$ \\
5 & $\left(17.8^{\circ},-3.8^{\circ}\right)$ & $\left(-14.1^{\circ}, 12.8^{\circ}\right)$ \\
6 & $\left(8.3^{\circ},-11.6^{\circ}\right)$ & $\left(-72.6^{\circ}, 6.0^{\circ}\right)$ \\
7 & $\left(-46.6^{\circ}, 30.2^{\circ}\right)$ & $\left(-7.7^{\circ}, 44.8^{\circ}\right)$ \\
8 & $\left(53.7^{\circ}, 18.6^{\circ}\right)$ & $\left(41.8^{\circ},-33.6^{\circ}\right)$ \\
\hline
\end{tabular}


It represents the sum of estimated variances of the $N_{\Omega}$ estimated coefficients $[34,35]$. When using the method described in [19], one obtains $\mathcal{C}=15.2$, whereas using the method described in the present paper, one obtains $\mathcal{C}=5.3$ : the estimation variance is thus reduced by about a factor 3 . This result confirms that by optimizing all the polarization states in illumination and analysis simultaneously, it is possible to enhance significantly the estimation precision of Mueller matrix coefficients. Of course, in some applications, it may be easier or cheaper to use a Stokes imager rather than a polarimetric system with full degrees of freedom. The advantage in terms of estimation precision that we have demonstrated has thus to be mitigated with implementation-related criteria to determine which is the best architecture for a given application.

\section{Estimation of Diagonal Mueller Matrices}

To validate our approach experimentally, we will now apply it to a real-world acquisition problem. We acquire 20 times the entire Mueller matrix of a wooden board and obtain the following average normalized Mueller matrix:

$$
\left[\begin{array}{cccc}
1.00 & -0.00 & -0.02 & 0.01 \\
-0.00 & 0.18 & 0.00 & 0.01 \\
-0.01 & 0.01 & -0.18 & 0.00 \\
0.01 & 0.01 & 0.00 & -0.09
\end{array}\right] .
$$

This matrix is almost diagonal, the nondiagonal coefficients being small. To characterize this type of wood, we thus only have to estimate the four coefficients $M_{00}, M_{11}, M_{22}$, and $M_{33}$. There are several sets of four PSG/PSA couples that lead to the optimal condition number of 2.2. One of them is given in Table 2 .

We implemented this set of states on our imager to measure the four coefficients using the method presented in Eq. (8). To reduce the influence of random fluctuations, we repeated the measure 20 times and obtained, on average, the four following coefficients:

$$
\begin{aligned}
& M_{00}=1 ; \quad M_{11}=0.18 ; \\
& M_{22}=-0.16 ; \quad M_{33}=-0.09 .
\end{aligned}
$$

We can see that there is a little difference between the estimated Mueller matrix and the real one. This error can be analyzed as due to the noise or as a bias due to a small nondiagonal coefficient in the Mueller

Table 2. Optimal States Used to Acquire the Four Images that Enable Extraction of the Four Relevant Coefficients of the Mueller Matrix of the Piece of Wood

\begin{tabular}{lcc}
\hline Image & $\left(\alpha_{s}, \varepsilon_{s}\right)$ & $\left(\alpha_{t}, \varepsilon_{t}\right)$ \\
\hline 1 & $\left(0^{\circ}, 0^{\circ}\right)$ & $\left(90^{\circ}, 0^{\circ}\right)$ \\
2 & $\left(45^{\circ}, 0^{\circ}\right)$ & $\left(45^{\circ}, 0^{\circ}\right)$ \\
3 & $\left(0^{\circ},-45^{\circ}\right)$ & $\left(0^{\circ}, 45^{\circ}\right)$ \\
4 & $\left(-22.5^{\circ},-17.62^{\circ}\right)$ & $\left(22.5^{\circ},-17.62^{\circ}\right)$ \\
\hline
\end{tabular}

Table 3. Standard Deviation (std) of the Coefficients Computed from the Acquisition of the Full Mueller Matrix or from the Acquisition of Four Optimized Images ${ }^{a}$

\begin{tabular}{lcc}
\hline & Full Mueller Matrix & Four Images \\
\hline $\operatorname{std}\left[M_{11}\right]$ & 0.02 & 0.01 \\
$\operatorname{std}\left[M_{22}\right]$ & 0.02 & 0.02 \\
$\operatorname{std}\left[M_{33}\right]$ & 0.02 & 0.02 \\
\hline
\end{tabular}

${ }^{a}$ Standard deviations are computed on 20 samples.

matrix. To confirm this, we have computed the standard deviation of the estimated coefficient computed from the four optimized images and the one of the coefficient coming from the full Mueller matrix and we have obtained the result presented in Table 3 .

We can see that the standard deviation on the coefficients is of the same order as the difference between the average values of the coefficients. Consequently, in this configuration, the discrepancy between the partial measures and the full ones [see Eq. (15)] is mainly due to measurement noise such as photon noise. The performance of estimation can thus be improved by increasing the integration time to acquire images with higher SNR, for example.

\section{Conclusion}

We have realized a polarimetric imaging system based on liquid-crystal modulators that allows generation and analysis of any polarization state on the Poincaré sphere. It is fully electronically controlled, and the maximal error on the generation of a polarization state is only $2.25^{\circ}$ in azimuth and ellipticity. This system is more versatile than standard Mueller imagers, and we have shown in several examples that it can perform better. First, this system makes it possible to implement the illumination and analysis states that optimize the discrimination between targets of interest and a background. Second, it can perform extraction of a subset of nonzero Mueller matrix coefficients with minimal error.

Of course, the demonstrated improvement in discrimination ability or estimation precision has to be mitigated with the cost and the ease of operation of other possible architectures. However, we are confident that the instrument and optimization approach proposed in this paper are efficient tools to investigate such applications of polarization imaging as remote sensing, imaging through turbid media, and industrial control.

The authors wish to thank Enric-Garcia Caurel, Antonello de Martino, and Razvigor Ossikovski for sharing with us their knowledge about Mueller matrix imager design and giving us access to their polarimetric measurement facilities. We also thank Jean-Michel Charbois, Vincent Devlaminck, and Patrick Terrier for useful advice on using LCVR in polarization imaging systems. Guillaume Anna's Ph.D. thesis is supported by the Délégation Générale pour l'Armement, MRIS domain IMAT. 


\section{References}

1. J. S. Tyo, M. P. Rowe, E. N. Pugh, and N. Engheta, "Target detection in optical scattering media by polarizationdifference imaging," Appl. Opt. 35, 1855-1870 (1996).

2. J. E. Solomon, "Polarization imaging," Appl. Opt. 20, 1537-1544 (1981).

3. R. Walraven, "Polarization imagery," Opt. Eng. 20, 14-18 (1981).

4. A. D. Martino, Y.-K. Kim, E. Garcia-Caurel, B. Laude, and B. Drévillon, "Optimized Mueller polarimeter with liquid crystals," Opt. Lett. 28, 616-618 (2003).

5. P. Lemaillet, S. Rivet, and B. L. Jeune, "Optimization of a snapshot Mueller matrix polarimeter," Opt. Lett. 33, 144-146 (2008).

6. P. A. Letnes, I. S. Nerbø, L. M. S. Aas, P. G. Ellingsen, and M. Kildemo, "Fast and optimal broad-band Stokes/Mueller polarimeter design by the use of a genetic algorithm," Opt. Express 18, 23095-23103 (2010).

7. J. S. Tyo, "Design of optimal polarimeters: maximization of signal-to-noise ratio and minimization of systematic error," Appl. Opt. 41, 619-630 (2002).

8. J. Zallat, S. Ainouz, and M. P. Stoll, "Optimal configurations for imaging polarimeters: impact of image noise and systematic errors." J. Opt. A 8, 807-814 (2006).

9. F. Goudail, "Noise minimization and equalization for Stokes polarimeters in the presence of signal-dependent Poisson shot noise," Opt. Lett. 34, 647-649 (2009).

10. E. Compain, S. Poirier, and B. Drevillon, "General and selfconsistent method for the calibration of polarization modulators, polarimeters, and Mueller-matrix ellipsometers," Appl. Opt. 38, 3490-3502 (1999).

11. G. W. Kattawar and M. J. Rakovic, "Virtues of Mueller matrix imaging for underwater target detection,” Appl. Opt. 38, 6431-6438 (1999).

12. K. M. Twietmeyer, R. A. Chipman, A. E. Elsner, Y. Zhao, and D. VanNasdale, "Mueller matrix retinal imager with optimized polarization conditions," Opt. Express 16, 21339-21354 (2008).

13. M.-R. Antonelli, A. Pierangelo, T. Novikova, P. Validire, A. Benali, B. Gayet, and A. De, Martino, "Mueller matrix imaging of human colon tissue for cancer diagnostics: how Monte Carlo modeling can help in the interpretation of experimental data," Opt. Express 18, 10200-10208 (2010).

14. D. G. Jones, D. H. Goldstein, and J. C. Spaulding, "Reflective and polarimetric characteristics of urban materials," Proc. SPIE 6240, 62400A (2006).

15. B. G. Hoover and J. S. Tyo, "Polarization components analysis for invariant discrimination," Appl. Opt. 46, 8364-8373 (2007).

16. G. Anna, F. Goudail, and D. Dolfi, "Polarimetric target detection in the presence of spatially fluctuating Mueller matrices," Opt. Lett. 36, 4590-4592 (2011).

17. G. Anna, F. Goudail, and D. Dolfi, "Optimal discrimination of multiple regions with an active polarimetric imager," Opt. Express 19, 25367-25378 (2011).
18. J. S. Tyo, S. J. Johnson, Z. Wang, and B. G. Hoover, "Designing partial Mueller matrix polarimeters," Proc. SPIE 7461 74610V (2009).

19. S. Savenkov, R. Muttiah, E. Oberemok, and A. Klimov, "Incomplete active polarimetry: measurement of the block-diagonal scattering matrix," J. Quant. Spectrosc. Radiat. Transfer 112, 1796-1802 (2011).

20. B. Laude-Boulesteix, A. De Martino, B. Drévillon, and L. Schwartz, "Mueller polarimetric imaging system with liquid crystals," Appl. Opt. 43, 2824-2832 (2004).

21. F. Goudail, P. Terrier, Y. Takakura, L. Bigué, F. Galland, and V. Devlaminck, "Target detection with a liquid crystalbased passive Stokes polarimeter," Appl. Opt. 43, 274-282 (2004).

22. A. Bénière, F. Goudail, M. Alouini, and D. Dolfi, "Design and experimental validation of a snapshot polarization contrast imager," Appl. Opt. 48, 5764-5773 (2009).

23. J. Liu and R. M. A. Azzam, "Polarization properties of cornercube retroreflectors: theory and experiment," Appl. Opt. 36, 1553-1559 (1997).

24. K. M. Twietmeyer and R. A. Chipman, "Optimization of Mueller matrix polarimeters in the presence of error sources," Opt. Express 16, 11589-11603 (2008).

25. M. Shribak, "Complete polarization state generator with one variable retarder and its application for fast and sensitive measuring of two-dimensional birefringence distribution," J. Opt. Soc. Am. A 28, 410-419 (2011).

26. A. B. Kostinski and W. M. Boerner, "On the polarimetric contrast optimization," IEEE Trans. Antennas Propagat. 35, 988-991 (1987)

27. M. Richert, X. Orlik, and A. De Martino, "Adapted polarization state contrast image," Opt. Express 17, 14199-14210 (2009)

28. F. Goudail and A. Bénière, "Optimization of the contrast in polarimetric scalar images," Opt. Lett. 34, 1471-1473 (2009).

29. F. Goudail, "Comparison of the maximal achievable contrast in scalar, Stokes and Mueller images," Opt. Lett. 35, 2600-2602 (2010).

30. J. S. Tyo, Z. Wang, S. J. Johnson, and B. G. Hoover, "Design and optimization of partial Mueller matrix polarimeters," Appl. Opt. 49, 2326-2333 (2010).

31. Q. Y. Duan, V. K. Gupta, and S. Sorooshian, "A shuffled complex evolution approach for effective and efficient global minimization," J. Optim. Theory Appl. 76, 501-521 (1993).

32. A. Ambirajan and D. C. Look, "Optimum angles for a polarimeter: part II,” Opt. Eng. 34, 1656-1658 (1995).

33. J. S. Tyo, "Noise equalization in Stokes parameter images obtained by use of variable-retardance polarimeters," Opt. Lett. 25, 1198-1200 (2000).

34. A. Papoulis, Probability, Random Variables and Stochastic Processes (McGraw-Hill, 1984).

35. F. Goudail and A. Bénière, "Estimation precision of the degree of linear polarization and of the angle of polarization in the presence of different sources of noise," Appl. Opt. 49, 683-693 (2010). 